\title{
Topological insulator based locally resonant phononic crystals: Wave propagation and acoustic band gaps
}

\section{Oral Oltulu, Sevket Simsek, Amirullah M. Mamedov \& Ekmel Ozbay}

To cite this article: Oral Oltulu, Sevket Simsek, Amirullah M. Mamedov \& Ekmel Ozbay (2016) Topological insulator based locally resonant phononic crystals: Wave propagation and acoustic band gaps, Ferroelectrics, 499:1, 123-129, DOI: 10.1080/00150193.2016.1173507

To link to this article: https://doi.org/10.1080/00150193.2016.1173507

曲 Published online: 31 May 2016.

Submit your article to this journal

Џ Article views: 216

Q View related articles $\widetilde{ }$

View Crossmark data $\nearrow$

Citing articles: 1 View citing articles 


\title{
Topological insulator based locally resonant phononic crystals: Wave propagation and acoustic band gaps
}

\author{
Oral Oltulu ${ }^{a}$, Sevket Simsek ${ }^{b}$, Amirullah M. Mamedov ${ }^{c, d}$, and Ekmel Ozbay ${ }^{d}$ \\ ${ }^{a}$ Department of Physics, Harran University, Sanlıurfa, Turkey; ${ }^{b}$ Department of Material Science and Engineering, \\ Faculty of Engineering, Hakkari University, Hakkari, Turkey; ${ }^{\mathrm{C}}$ Nanotechnology Research Center (NANOTAM), \\ Bilkent University, Ankara, Turkey; International Scientific Center, Baku State University, Baku, Azerbaijan
}

\begin{abstract}
In the present work the acoustic band structure of a two-dimensional phononic crystal (PC) containing an organic ferroelectric (PVDFpolyvinylidene fluoride) and topological insulator $\left(\mathrm{Bi}_{2} \mathrm{Te}_{3}\right)$ were investigated by the plane-wave-expansion (PWE) method. Twodimensional PC with square lattices composed of $\mathrm{Bi}_{2} \mathrm{Te}_{3}$ cylindrical rods embedded in the PVDF matrix are studied to find the existence of stop bands for the waves of certain energy. Phononic band diagram $\omega=\omega(\mathbf{k})$ for a $2 \mathrm{D}$ PC along the $\Gamma-\mathrm{X}-\mathrm{M}-\Gamma$ path in the square Brillouin zone show four stop bands in the frequency range $0.01-8.0 \mathrm{kHz}$.
\end{abstract}

\section{ARTICLE HISTORY}

Received 28 June 2015

Accepted 12 November 2015

\section{KEYWORDS}

Phononic crystals; acoustic band gaps; topological insulator; wave propagation

\section{Introduction}

There has been a great deal of interest in recent years in the study of two-dimensional (2D), periodic arrangements of inclusions inside a viscoelastic material or a fluid, so-called phononic crystals $[1,2]$. Phononic crystals (PCs) are composite materials that are artificially manufactured structures with special properties regarding wave propagation, known as acoustic metamaterials. The most interesting aspect of such materials arises from the presence of phononic band gaps (PBGs). The band gap in a phononic crystal is caused by a periodic variation in the density and changes of the speed of sound in the crystal. The band gaps exist in a certain range of frequencies where acoustic/elastic waves propagation is forbidden. In such a situation, the phononic crystal behaves as a perfect acoustic mirror and an incident wave is perfectly reflected outside for the rejected frequencies [3].

The band structure of a phononic crystal can be controlled by changing the geometry of the inclusions [4], the volume fraction of inclusions [5], by varying lattice spacing and lattice topology [6], or by varying the mechanical characteristics of the constitutive materials that change through the application of external stimuli [7]. However, some authors have also studied and analyzed the effects of piezoelectricity on the dispersion curves and they show that the change is negligible for small filling fractions, but significant for large ones $[8,9]$.

Bismuth-based binary compounds such as bismuth telluride $\left(\mathrm{Bi}_{2} \mathrm{Te}_{3}\right)$ is a narrow band gap semiconductor and has been known for a long time to be excellent thermoelectric

CONTACT Oral Oltulu oltulu@harran.edu.tr

Color versions of one or more of the figures in the article can be found online at www.tandfonline.com/gfer. 
materials and an acoustic topological insulator, which is also one of the most commonly studied topological material to date [10]. The concept of topologically nontrivial band structures has recently led to the emergence of the field of "topological photonics" and then, the concept is extended to acoustics by designing a phononic crystal [11]. These phononic metamaterials may allow us to have access to a much wider range of material properties, since their topological characteristics can survive to high temperatures. On the other hand, polyvinylidene fluoride (PVDF) is a well- known material for its toughness, and stability, as well as its ability to withstand long-term exposure to ultraviolet radiation. The major advantage of using polymeric materials is that they have low weight, low thermal conductivity, high heat resistance, and mechanical flexibility.

This paper theoretically and numerically investigates the propagation characteristics and the band structure of longitudinal waves propagating in a $2 \mathrm{D}$ phononic crystal composed of $\mathrm{Bi}_{2} \mathrm{Te}_{3}$ cylinders embedded in a PVDF matrix. More explicitly, we study the $2 \mathrm{D}$ composites by solving the basic acoustic wave equation and use Bloch wave analysis to identify the band gaps. The results obtained are based on the plane wave expansion method.

\section{Model and the calculation method}

Here we focus on the P-wave (pressure wave) or acoustic wave propagation through a periodic array of cylindrical scatterers. Although one of the media is a solid medium, we will assume the matrix as a fluid medium to take the advantage of solid/fluid approximation. Such approximation is reasonable if we only consider that the waves propagate as longitudinal waves by means of adiabatic compression and decompression. The major difference between the approximations will mainly be observed for higher bands. In other words, this approximation can yield acceptable results for lower bands. Assuming a homogeneous and isotropic medium, we can separate the wave modes and use methods based on acoustic wave theory. Among them, the PWE method in the frequency domain [12], and the FDTD method in time domain [13] are the two that are used most widely. We will use the PWE method because of its simplicity. The advantage of the PWE method is that it must neglect the transverse mode existing in solid and simply treat the solid as an artificial "fluid." It must be noted that one of the advantages of PVDF like materials is that they can be fluidprocessed as a melt. The considered system is a $2 \mathrm{D}$ phononic crystal composed of $\mathrm{Bi}_{2} \mathrm{Te}_{3}$ inclusions in a PVDF host in a square lattice. A square lattice formed of cylindrical rods has a lattice constant, $\mathrm{a}=0.05 \mathrm{~m}$ and the scatterers' radius, $\mathrm{r}_{0}=0.025 \mathrm{~m}$. The calculation model of the studied system and its unit cell is shown in Figure 1.

The material properties are $\rho_{1}=1780 \mathrm{~kg} / \mathrm{m}^{3}$ and $c_{\mathrm{L} 1}=1300 \mathrm{~m} / \mathrm{s}$ for PVDF, and $\rho_{2}=$ $7858 \mathrm{~kg} / \mathrm{m}^{3}$ and $\mathrm{c}_{\mathrm{L} 1}=2951 \mathrm{~m} / \mathrm{s}$ for $\mathrm{Bi}_{2} \mathrm{Te}_{3}$. For the PWE method the solid matrix is considered as a fluid but with the material parameters of actual PVDF. Under the assumptions we made, the acoustic wave equation can be written as

$$
\left(C_{11}\right)^{-1} \frac{\partial^{2} p}{\partial t^{2}}=\vec{\nabla} \cdot\left(\rho^{-1} \nabla p\right)
$$

where $\mathrm{p}$ is the pressure wave, $\mathrm{C}_{11}$ is the relevant elastic constant and $\rho$ is the mass density. The PWE expansion method has been applied to acoustic wave equation in calculating the band gap of the system. 


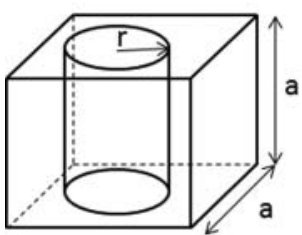

(a)

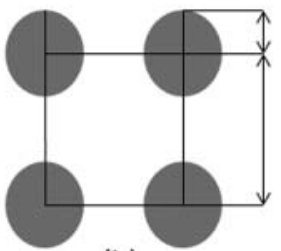

(b)
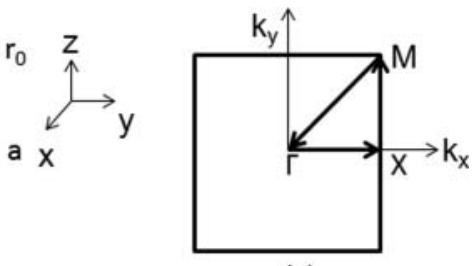

(c)

Figure 1. Unit cell of a square lattice two dimensional phononic crystal (a). Transverse cross-section of a representative 2-D phononic crystal (b) and Brillouin zone of 2-D square lattice PCs (c).

\section{Results and discussion}

The computational model based on the PWE method and the physical parameters of the system have been given in the above section. The numerical results for the band structure are expressed in terms of the normalized or reduced frequency $\Omega$, which is an expression of $\left(\omega a / 2 \pi c_{l}\right)$ and it ranges from 0 to 3.0. Figure 2 shows the calculated band structures of the $2 \mathrm{D}$ phononic crystal given in Figure 1. It is seen in Figure 2 that there exists complete acoustic band gaps (indicated by shaded region) for the assumed model around 0.4 to 2.8. The complete band gap along the right-handed triangle $\Gamma-\mathrm{X}-\mathrm{M}-\Gamma$ with vertices at $\Gamma=(0,0), \times=$ $(\pi / \mathrm{a}, 0)$ and $\mathrm{M}=(\pi / \mathrm{a}, \pi / \mathrm{a})$ show us that the entire angular range of the wave propagation direction, while other directions are not forbidden within a certain range of frequencies. The density of states (DOS) as a function of frequency is also computed and the figure in the right side panel shows the corresponding phononic density of states as a function of frequency. The DOS were calculated by sampling k-points in the two dimensional Brillouin zone (BZ). We found the DOS as a function of frequency $\omega$ by doing a sum of all the bands over all the frequencies.

The dispersion relation, $\omega(\mathbf{k})$, for the first and second bands were calculated. Like the band structure construction, we used the frequency-domain PWE method to create the equi-frequency contours (EFCs). They are illustrated in Figure 3(a) and Figure 4(a),

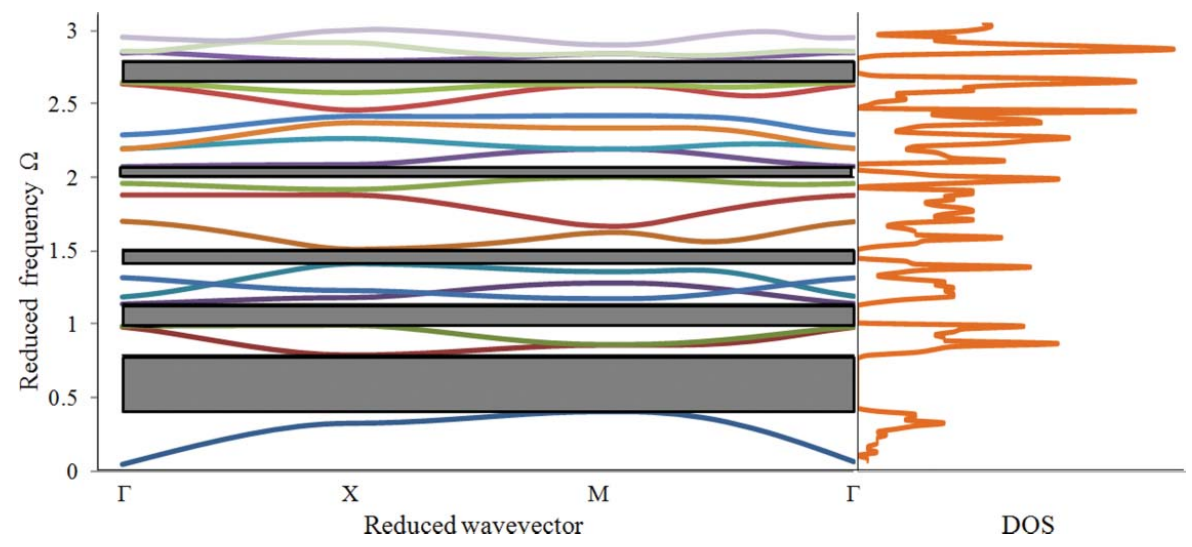

Figure 2. The band structure of the studied 2D PVDF/Bi ${ }_{2} \mathrm{Te}_{3}$ phononic crystal calculated in the first Brillouin zone. 
respectively. The plots provide a better understanding of how such dispersion influences acoustic velocity. Moreover, the contour plots of the associated dispersion surfaces are shown in Figure 3(b) and Figure 4(b). The EFCs are at the intersection of the 3D dispersion curves with a horizontal plane and they provide an essential source of information. By considering the acoustical wave vector and the group velocity as local parameters, the shape of the EFCs can be used accurately to account for anisotropy. Figure 3(b) shows EFCs in reduced $\mathbf{k}$ space and they are mostly circular around the high symmetry point. Circular dispersion in reduced $\mathbf{k}$ space means that the medium is an isotropic medium and the wavevector of the acoustic wave and the group velocity are parallel for any propagation direction. Therefore, in the material the velocity of the acoustic wave does not depend on the direction that the wave travels. As can be seen, the radius of the EFCs clearly increases as the frequency increases.

The equi-frequency surfaces, (EFSs), of the incident wave along the $\Gamma-\mathrm{X}-\mathrm{M}-\Gamma$ direction are shown in Figure 4(b). Figure 4(b) shows that local curvature of the EFCs deviates from circular symmetry. An equi-frequency plot of the 2 nd band, which is a little bit more complicated than the 1st band, exhibits a square-like contour. The case of a non-circular EFCs, however, indicates anisotropic behavior. On the other hand, a square like contour also reflects some symmetry in a particular direction. This could especially be advantageous and
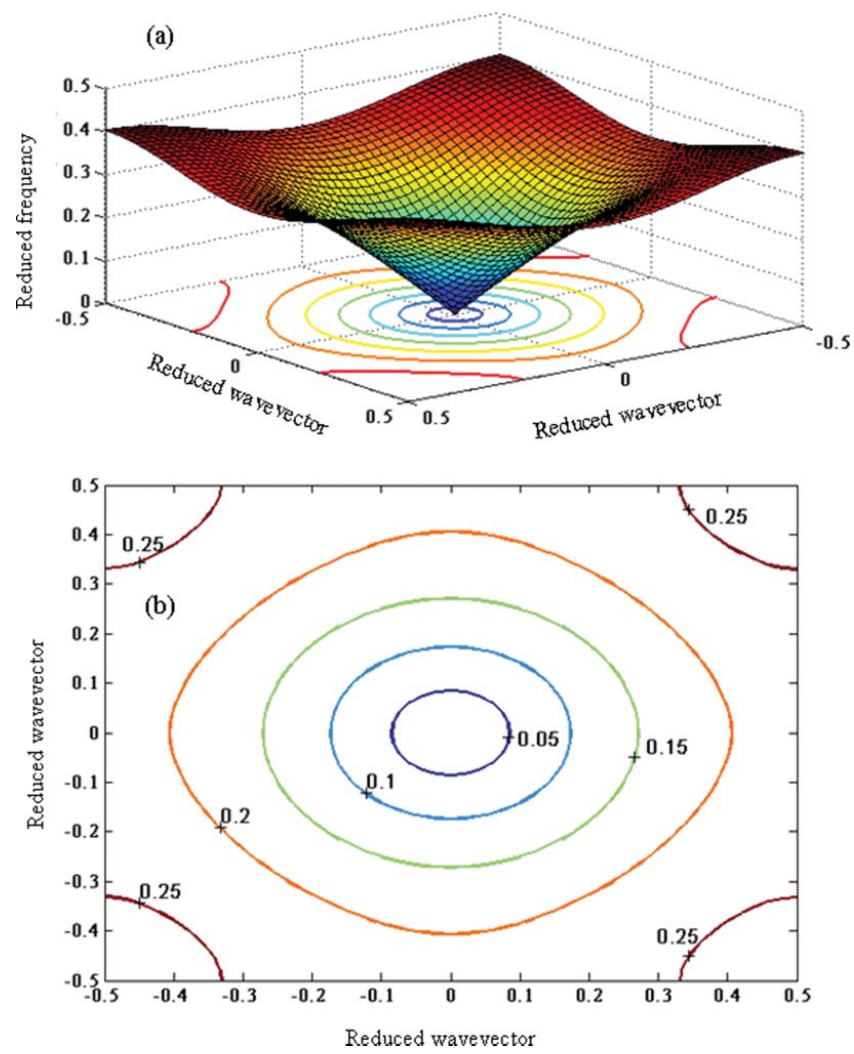

Figure 3. The dispersion, $\omega(\mathbf{k})$, relation (band-diagram) for the first bands of the square phononic crystal., calculated for all k-vectors in the first Brillouin zone. (b) The equi-frequency contours for the first bands zone. 

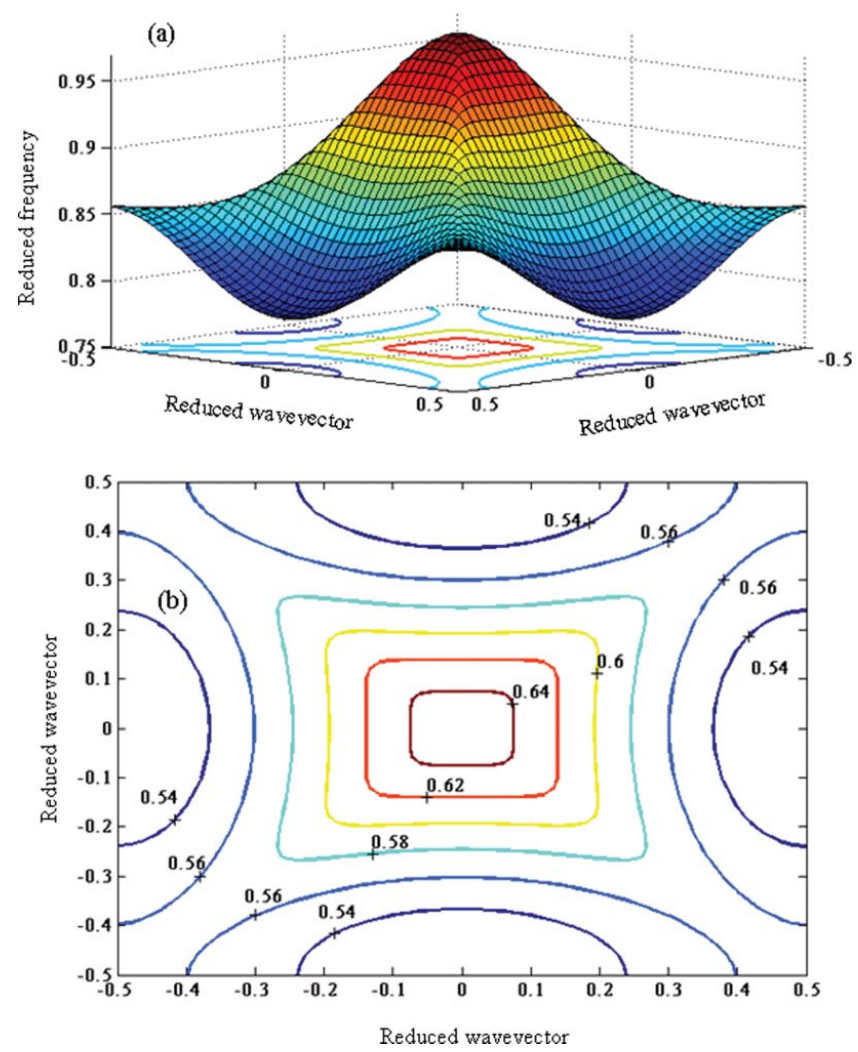

Figure 4. The dispersion, $\omega(\mathbf{k})$, relation (band-diagram) for the first bands of the square phononic crystal, calculated for all k-vectors in the first Brillouin zone. (b) The equi-frequency contours for the first bands zone.

even be more effective if the self collimation property of the phononic crystal is considered. It must be noted that the group velocity $\mathrm{V}_{\mathrm{g}}$ is normal to the EFC but not collinear to the wave vector $\mathbf{k}$. At each frequency, the energy flow direction is given by the normal to the $\mathrm{EFC}$, and is in the direction of the maximum rate of change of frequencies. The calculated contours also allow us to analyze whether the phononic crystal can have negative refraction or not, depending on the sign of the dispersion slope. From the EFC plots above, we can see that the radius of the circle increases with frequency, and we can conclude that the dispersion slope is positive.

The unusually large angles $\left(\sim 180^{\circ}\right)$ between the phase and group velocities of acoustic waves lead to many unusual wave phenomena observed at crystal-air or crystal-isotropic medium interfaces. In order to understand the dynamics of wave propagation, the concept of group velocity may be useful from the viewpoint of energy transportation. The direct calculation of the derivative of the dispersion relation calculated numerically is not always convenient and can give error. Thus, by using the results of [14-16] the group velocity can be always calculated more accurately, irrespective of the number of points in the dispersion curve. Therefore, we have calculated the group velocity along the high-symmetry directions $\Gamma-\mathrm{X}-\mathrm{M}-\Gamma$ of the BZ as shown in Figure 5. From Figure 5, it is evident that the components of the group velocity versus the high symmetry direction $(\Gamma-\mathrm{X}-\mathrm{M})$ vary over wide limits. The 


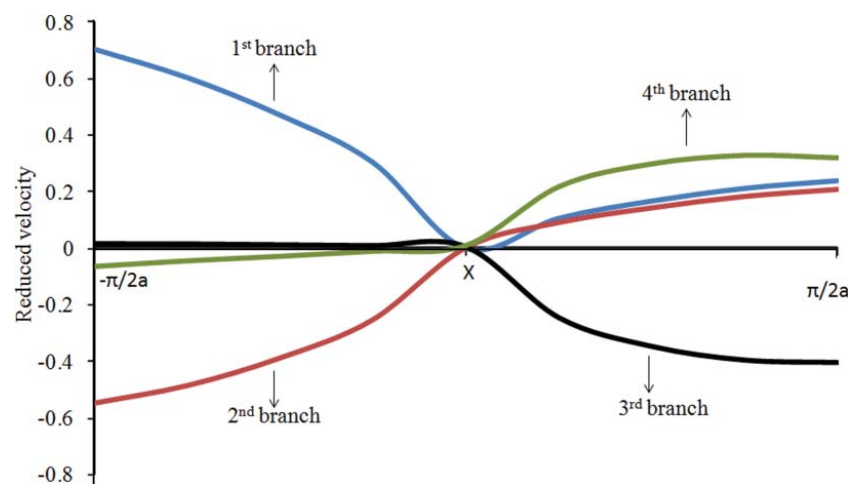

Figure 5. The first four branch group velocity $\partial \omega / \partial \mathrm{k}$ calculated along high symmetry directions of the first irreducible Brillouin zone.

dependences $V_{g}$, which describe the wave packets of localized modes of any order, exhibit (generally) a maximum at certain propagation constants. This means that the dispersion of group velocity can be positive, negative or zero $[15,16]$. It is worth mentioning that the group velocity of the waves is zero at the high symmetric point $\mathrm{X}$ meaning that there is no energy transfer at this point.

\section{Conclusion}

In this paper, we initially discussed the band structure of two dimensional phononic crystal composed of a topological material embedded in a polymer host. We use the plain wave expansion method to calculate band gaps in the acoustic band structure of the locally resonant phononic crystal. The results of this study illustrate that the width of the first band decreases and more phononic stop bands appear under the assumption of the fluid/solid approach. The findings of this paper may be useful to the improvements in the design of acoustic phononic crystals and they provide some perspectives in a way for designing acoustic filters or insulators.

\section{Acknowledgments}

This work is supported by the projects DPT-HAMIT, DPT-FOTON, and NATO-SET-193 as well as TUBITAK under the project nos., 113E331, 109A015, and 109E301. One of the authors (Ekmel Ozbay) also acknowledges partial support from the Turkish Academy of Sciences.

\section{References}

1. C. M. Soukoulis, Photonic Band Gaps and Localization. New York: Plenum Press (1993).

2. J. D. Joannopoulos, R. D. Meade and J. N. Winn, Photonic Crystals. New York: Princeton Univ. Press (1995).

3. Y. Pennec, B. Djafari-Rouhani, H. Larabi, J. Vasseur and A. C. Hladky-Hennion, Phononic crystals and manipulation of sound. Phys. Status Solidi 6(9), 2080-2085 (2009).

4. C. Goffaux and J. P. Vigneron, Theoretical study of a tunable phononic band gap system. Phys. Rev. B 64(7), 075118 (2001). 
5. R. Sainidou, N. Stefanou, A. Modinos, Formation of absolute frequency gaps in three-dimensional solid phononic crystals. Phys. Rev. B 66, 212301 (2002).

6. T. Gorishnyy, C. K. Ullal, M. Maldovan, G. Fytas, E. L. Thomas, Hypersonic phononic crystals. Phys. Rev. Lett. 94, 115501 (2005).

7. J. Baumgartl, M. Zvyagolskaya, C. Bechinger, Tailoring of phononic band structures in colloidal crystals. Phys Rev Lett. 99(20), 205503 (2007).

8. Z. Hou, F. Wu and Y. Liu, Phononic crystals containing piezoelectric material, Solid State Commun. Solid State Commun. 130, 745-749 (2004).

9. Y. Z. Wang, F. M. Li, Y. S. Wang, K. Kishimoto, W. H. Huang, Tuning of band gaps for a twodimensional piezoelectric phononic crystal with a rectangular lattice. Acta Mechanica Sinica 25, 65-71 (2009).

10. J. Moore, Topological insulators: The next generation. Nature Physics 5, 378-380 (2009).

11. Z. Yang, F. Gao, X. Shi, X. Lin, Z. Gao, Y. Chong, B. Zhang, Topological Acoustics. Phys. Rev. Lett. 114, 114301 (2015).

12. M. S. Kushwaha, P. Halevi, L. Dobrzynski, B. Djafari-Rouhani, Acoustic band structure of periodic elastic composites. Phys Rev Lett. 71(13), 2022-2025 (1993).

13. Y. Tanaka, Y. Tomoyasu and S. Tamura, Band structure of acoustic waves in phononic lattices: Two-dimensional composites with large acoustic mismatch. Phys. Rev. B 62(11), 7387-7392 (2000).

14. A. Srivastava, Elastic metamaterials and dynamic homogenization: a review. Int. J. of Smart and Nano Materials 6(1), 41-60 (2015).

15. V. A. Burov, V. B. Voloshinov, K. V. Dmitriev, N. V. Polikarpova, Acoustic waves in metamaterials, crystals, and anomalously refracting structures. Phys. Usp. 181(11), 1205-1211 (2011).

16. R. V. Craster, S. Guenneau, Acoustic Metamaterials: Negative Refraction, Imaging, Lensing and Cloaking. New York: Springer Series in Materials Science (2013). 